\title{
Clinical and Pathological Aspects of HIV Infection in Adults
}

\author{
SEBASTIAN B. LUCAS, FRCPath(Lond) \\ UMDS, Department of Clinical Histopathology, St. \\ Thomas' Hospital, London, United Kingdom
}

\begin{abstract}
This manuscript reviews the beginning of the appearance of the AIDS syndrome in mid-1980 in homosexuals in the U.S.A. which had presented with Kaposi's sarcoma, Pneumocystis carinii infections, low blood T-helper cells (CD4+), and reversal of CD4/CD8 T-cells ratio. Later the disease appeared to be transmitted by sexual intercourse and blood products and was recognized in other parts of the world. Originally termed "HTLV-III". it was renamed "HIV". Modes of transmission of HIV. its epidemiology, pathophysiology of HIVIAIDS, case definitions of AIDS. AIDS-defining conditions. clinico-pathological features of HIV disease, and the impact of therapy on HIV disease are discussed.
\end{abstract}

Keywords: HIV.Pathological aspects, Transmission.

\section{Introduction}

In mid-1981, tw̌o reports emanated from the Centres for Disease Control (CDC) in Atlanta, Georgia, USA concerning unusual disease syndromes among a small number of previously healthy white Americans. They had presented with Kaposi's sarcoma (KS, a previously very rare vascular tumour), or Pneumocystis carinii pneumonia (PCP), a rare infection seen previously in malnourished infants or adults immunosuppressed by malignancy or iatrogenicallyll-U. All the patients were homosexual, and laboratory tests demonstrated profound cellular immunodeficiency, with low 'blood T-helper cells (CD4+) and reversed CD4/CD8 T-cell ratios. The mortality was high and soon, large numbers of males with similar syndromes were being registered. The original opportunistic diseases of PCP and KS were the forerunners of many diverse infections and tumours that developed in these patients.

Correspondence\& reprint requests to: Prof. Sebastian Lucas, UMDS, Department of Clinical Histopathology, 51. Thomas' Hospital,London SEI. United Kingdom. Presented at the International Symposiumon Pathology and Laboratory Medicine, 15-17 November 1997. Jeddah, Saudi Arabia.

Acceptedfor publication: 16March 1998. Received: 13January 1998. 
Within three years there were four significant developments. First, the syndrome which became known as acquired immunodeficiency syndrome (AIDS) could affect anyone--females, young children, heterosexual males, haemophiliacs, and intravenous drug users. Secondly, the disease was quickly recognized in many parts of the world, including large numbers of patients in east and central Africa as well as in the industrialized nations. Thirdly, because the epidemiology pointed to an infectious agent that could be transmitted by blood products and by sexual intercourse. By 1984 intensive research identified the cause as an RNA retrovirus analogous to the human T-celllymphoma/leukaemia viruses. Originally termed "HTLV-III", it was soon renamed "human immunodeficiency virus (HIV)". It became evident that a slightly different HIV was prevalent in west Africa: the major type, present globally, is termed "HIV-1" and the other much less common one, "HIV-2"[Jl. Dual infections are common in west Africa. Fourthly, a blood test was developed that could identify those infected but not symptomatic by detecting antibodies to HIV.

Epidemiology of HIV infection: HIV infection is prevalent in every country on the globe but at vastly different rates. The latest predictions are that by the end of 1997 , 30.6 million people will be HIV-infected and that the total will rise by the year 2000 to 40 million. Two-thirds of them live in sub-Saharan Africa, and one-fifth in Asia. In 1997 alone, some 2.3 million people will have died of AIDS. Ninety percent (90\%) of those infected reside in the developing resource-poor world, and $40 \%$ are women. Some 2.7 million children have already died of HIV disease and 1.1 million are currently infected. Each day some 16,000 previously uninfected persons are infected with HIV. Nine out of 10 infected people do not know they are infected (and can transmit the infection). Not surprisingly, in the countries most severely affected, life expectancy is falling and infant mortality rates have risen dramatically (UNAIDS Report, November 1997) (Table 1).

TABLE I. Modes of transmission of HIV

Heterosexual intercourse (male to female and female to male)

Homosexual ano-rectal intercourse.

Perinatal, mother to infant (during pregnancy, delivery, and by breast feeding).

Blood products (whole blood and concentrates).

Percutaneous injury and mucosal contamination in health care workers (rare).

Pathophysiology of HIV/AIDS[4]: There are three phases of the host-virus interaction following infection: 1) infection, 2) clinical latency, 3) severe cellular immunosuppression and clinical illness.

The RNA virus enters across mucosal (genital) surfaces or through inoculation, and binds to cells which possess the CD4 receptor. These include T-helper cells, macrophages, and Langerhans cells. Once inside the cell, reverse transcriptase enzyme produces a DNA copy of the virus which then integrates into the host cell genome. It can remain in a latent state or produce new virions which are exported and infect other CD4+ T-cells. Productive infection is also cytotoxic to the host cell by the process of 
apoptosis. The dynamics of virus/T-cell interaction are of great complexity and incompletely understood. In summary, there is an initial widespread dissemination of HIV throughout the lymphoid system after infection, with viraemia and a transient drop in blood CD4+ T-cell counts. This coincides with the seroconversion illness that about half of those infected develop and antibodies to HIV appear--within 6-12 weeks. The viraemia is then controlled by the development of cytotoxic lymphocytes (CTL).

During the latent period which lasts for several years, HIV infection is destroying Tcells at a rate $(2 \times 10 \%$ day $)$ faster than they can be replenished. Whilst viraemia is low or undetectable, virus is present productively and latently in the lymphoid system. However, the immune system is still able to defend the host against opportunistic diseases. Early during this phase, many patients develop a transient widespread lymphadenopathy, termed "persistent generalized lymphadenopathy" (POL). In the final phase of severe cellular immunosuppression, the cell-mediated immune system collapses and a variety of infections and tumours (i.e., AIDS) that are normally kept at bay develop. Death from combinations of these opportunists and from wasting followsl-1. Time from AIDS diagnosis to death varies from six to 18 months, but is increasing with new therapy (see below). Time from HIV infection to death is now $>10$ years for adults with access to good treatments.

Case definitions of AIDS: Before the HIV serotest became available, AIDS was originally defined by the presence of certain opportunistic diseases in patients without known causes of immunosuppression. In 1987 and subsequently in 1992, the CDC codified HIV and AIDS for the purposes of better surveillancels-"l, Whilst it may be ideal to count and register people with known HIV infection--in order to comprehend the extent of and changes in HIV in the community--there are practical and ethical problems in doing so. Also, many countries do not have ready access to HIV tests. It proved simpler to register patients who have positive HIV serology and one or more of a set of conditions that indicated significant immunodeficiency. These are diseases that are much more frequent and clinically severe in HIV-infected people compared with nonHIV-infected, and often have different clinico-pathological patterns.

Many other diseases are strongly associated with HIV infection and immunodeficiency. They include: visceral leishmaniasis, microsporidiosis, bacillary angiomatosis, nocardiosis, squamous carcinoma of the conjunctiva, and anal intra-epithelial neoplasia" (Tạble 2).

Clinico-pathological features of HIV disease: The seroconversion illness resembles infectious mononucleosis with fever, pharyngitis, skin rash, and lymph gland enlargement. An aseptic meningitis may occur and in a few patients, the transient immunodeficiency may be severe enough to predispose to pneumocystosis,

During the clinically latent phase POL may develop, and the lymphoid tissue in the parotid gland may expand similarly causing bilateral salivary gland swelling. Histologically, the nodes show a marked and characteristic irregular B-cell follicle hyperplasia with abundant tingible body macrophages and thin T-cell mantles. HIV is detectable in the follicle dendritic cells within the follicles, and their architecture is subsequent. 
During the clinically latent phase POL may develop, and the lymphoid tissue in the parotid gland may expand similarly causing bilateral salivary gland swelling. Histologically, the nodes show a marked and characteristic irregular B-cell follicle hyperplasia with abundant tingible body macrophages and thin T-cell mantles. HIV is detectable in the follicle dendritic cells within the follicles, and their architecture is subsequently destroyed. In the parotid gland, there is often a cystic dilatation of the ducts (lymphoepithelial cyst).

TABLE: 2. AIDS--defining conditions.

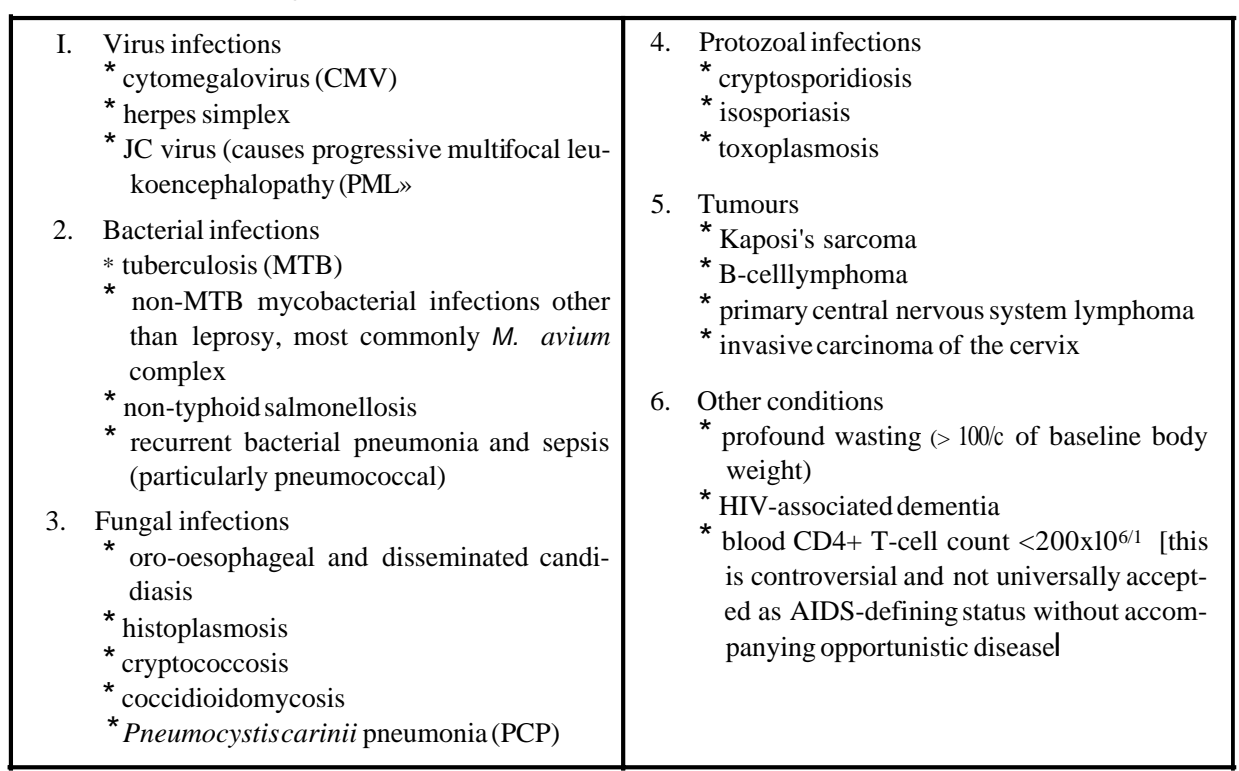

The many stages of HIV disease develop when depressed host cell-mediated immunity permits acquired and latent infections to develop. We know that there is a hierarchy of infections over time, with the more virulent agents causing disease earlier than those less virulent. The most virulent infection is M. tuberculosis, so in areas where it is highly prevalent, it presents an early and continuous problem in the management of HIV positive patientsl'il. In contrast, PCP is rare until the blood CD4 count drops below $200 \times 10^{6} / 1$. Severe CMV infection and M. avium complex occur even later, usually with CD4 counts $<50 \times 10^{6 / 1}$.

Community-acquired bacterial infections--Streptococcus pneumoniae and Salmonella enteritidis and Typhimurium-ese common recurrent infections causing bacteraemias that clinically present non-specifically with fever and diarrhoeal'", Tuberculosis may present early as a pulmonary disease similar to that in HIV-uninfected people, and is readily treatable. However, as immunosuppression worsens, tuberculosis becomes more disseminated with involvement of nodes, spleen, liver, marrow, lungs, and meninges. The histopathology, in turn, changes from the classical caseating granuloma with scanty acid-fast bacilli detectable, to a non-reactive highly necrotic lesion characterised by ab- 
sence of acivated macrophages and vast numbers of bacilli. At this stage, the infection has a high mortality even with chemotherapy.

Lymphoma and other tumoursllvl: HIV-associated lymphoma is mainly B-cell in origin and either Burkitt lymphoma or large cell in histological type. The pathogenesis is complex, but EBV is often involved. Typical is the location outside the lymph nodes-brain (see below), gut, mouth, anus, lung, and retroperitoneum are common sites. They develop usually late in the course of HIV disease and are often refratory to chemotherapy.

Kaposi's sarcoma (KS)--once an enigma in its aetiology and status as a tumour--is emerging as an opportunistic disease related to an infection, and its histogenesis is a reactive (probably, rather than neoplastic) proliferation of endothelial cells. The recent discovery of human herpes virus type 8 ,as a KS-associated virus, has stimulated much epidemiological study into its transmission!11]. KS affects the skin, mucosae, and lung, producing plaques and nodules. A major consequence is tissue oedema in the skin and soft tissues, and pulmonary oedema in the lungs which is a common cause of death.

Squamous in situ and invasive carcinomas of the cervis, anus, and conjunctiva are in-. creased in incidence in HIV-positive people. The pathogenesis appears to involve human papilloma virus infections, usually types HPV-16 and 18 and, in the case of the eye, UV light. Interestingly, in the cervix and anus, whilst the frequency of in situ lesions is relatively high, actual invasive carcinoma is much less common than would be expected. Whether this non-sequence is a real biological phenomenon or merely follows from the early death of the patient will await further follow-up as patients with HIV live longer with modern therapies.

eNS disease: The brain is affected at some time in about half of all HIV-infected patientsl 12]. The main pathologies are:

* direct HIV infection of the brain--HIV encephalitis

* opportunistic infections--CMV, toxoplasma, cryptococcosis, MTB meningitis

* high-grade Bl-celllymphoma

* vacuolar myelopathy of the spinal cord

HIV encephalitis is the main underlying pathology of the HIV-associated dementia clinical syndrome, though the correlation of clinico-imaging features with autopsy pathology is not exact. Histologically it includes the pathognomonic multinucleate giant cell encephalitis and leuco-encephalopathy. Clusters of microglial cells (nodules) are found throughout the neuraxis, particularly in the white matter. Multinucleate giant forms of the same macrophage-derived cells are found in small or large numbers, associated with local myelin damage. They contain HIV and are often productive of new virions. The pathogenesis of this encephalitis may involve the secretion of cytokines such as tumour necrosis factors (TNF) by the giant cells and microglia, with impairment of neural transmission within the brain and cord.

CMV encephalitis and ventriculitis is a cause of widespread CNS dysfunction and is frequent as a terminal condition. Toxoplasma encephalitis (TE) presents most commonly as focal neurological lesions, less often as a diffuse encephalitis, and is difficult to 
distinguish from lymphoma on clinical and imaging criteria. The frequency of TE in HIV -positive patients depends on the prevalence of Toxoplasma gondii latent infection in the population; this may range from $>90 \%$ of adults where uncooked meat is commonly eaten (Paris; west Africa), to $10 \%$ in dry southern Africa. Cerebral lymphoma is another common near-terminal condition where the tumour cells appear to originate in the walls of the intracerebral arteries before invading and destroying the parenchyma. These B-cell tumours all contain Epstein-Barr virus (EBV) in the nucleus, indicating a role in carcinogenesis. Because of the problem in diagnosing lymphoma, PCR to detect IBV in the cerebrospinal fluid may be used; empirical anti-toxoplasma therapy is given to exclude that infection prior to investigating lymphoma by brain biopsy or empirical therapy.

Renal disease in HIV infection: The kidney can be involved in any of the disseminated opportunistic infections such as cryptococcosis, bacterial pyaemia, miliary tuberculois and CMV[13]. However, there is also an HIV-associated nephropathy (HIVAN) that is characterised by marked tubular dilatation, interstitial nephritis, and segmental glomerulosclerosis. Clinically it causes rapid renal failure. HIV may be demonstrated within glomeruli. The striking feature of HIV AN is its almost exclusive predilection for African patients, both in Africa and in industrialized countries. This suggests that there must be a genetic predisposition to HIV AN, perhaps mediated through the HLA system.

Impact of therapy on HIV disease: Modern therapy is directed along four paths:

1. anti-HIVagents[14]

2. prophylaxis against common severe opportunistic infections

3. treatment of opportunistic infections and tumours

4. general supportive measures, e.g., nutritional support

The anti-HIV drugs include reverse transcriptase inhibitors (RTI) such as azidothymidine (AZT) and the more recent protease inhibitors. Combinations of the various agents are commonly used. Their action is to reduce the HIV viral load in tissues and blood and reduce the killing rate of $\mathrm{CD} 4+\mathrm{T}$-cells, thereby raising the blood CD4 counts. There is a definite impact on morbidity and mortality with patients in extremis demonstrating dramatic clinical improvement. Several opportunistic diseases regress through this anti-immunosuppressive effect alone. How long such agents will remain effective given the mutation rate of HIV and the tendency towards drug resistance is unknown at present. Pathologically, there are many side effects of the RTI drugs including marrow suppression with cytopaenias and fatty change of the liver which may be fatal.

Prophylactic therapy usually includes the effective anti-Pneumocystis agent cotrimoxazole, and accounts for a reduced prevalence of PCP among HIV-infected patients in Europe. It also has an anti-Toxoplasma effect and may explain the relative infrequency of toxoplasma encephalitis among those who have latent infection (as demonstrated serologically).

The treatment of specific opportunistic diseases involves a wide range of potent drugs with the expected occurrence of multi-organ toxicities. Given the importance of mycobacterial infections-vtuberculosis and non-MTB species--in HIV disease, toxicity from 
rifampicin, and other anti-mycobacterial agents is particularly important. Liver damage (hepatitis) is frequent. Those countries that use thiacetazone as ananti-M'I'B drug have discovered significant rates of erythema multiforme drug reactions with some deaths from toxic epidermal necrolysisl15].

In conclusion, HIV disease present a paradigm of what occurs when the cellmediated immune system is progressively destroyed. An ever-expanding range of relatively non-virulent pathogens invade and damage the body, in addition to the expected virulent agents. It is likely that modern chemotherapy will prolong the life of HIVinfected persons by ever improving anti-HIV and anti-opportunist chemotherapy--but ar increasing cost and with increasing prevalence of associated toxic side effects.

References

[ I] Centers for Disease Control and Prevention. Pneumocystis carinii pneumonia--Los Angeles. MMWR 1981;36: 250-252.

[2] Centers for Disease Control and Prevention. Kaposi's sarcoma and Pneumocysstis carinii pneumonia among homosexual men--New York City and California. MMWR 198J; 30: 305-308.

[3] Barre-Slnoussi F. HIV as the cause of AIDS. Lancet 1996;348: 31-35.

[4] Feinberg MB. Changing the natural history of HIV disease. Lancet 1996;348: 239-246.

[5] Lucas SB, De Cock KM, Hounnou A, Peacock CS, Diomande M, Honde M, Beaumel A, Kestens L, Kadio A. A contribution of tuberculosis to slim disease in Africa. Br Med J 1994;308: 1531-1533.

[6] Centers for Disease Control and Prevention. Revision of the CDC surveillance case definition for AIDS. MMWR 1987;36: IS-15S.

[7] Centers for Disease Control and Prevention. 1993 revised classification system for HIV infection and expanded surveillance case definition for AIDS among adolescents and adults. MMWR 1992; 41[RR17]: 1-19.

[8] Lucas SB, Hounnou A, Peacock CS, Beaumel A, Djomand G, N'Gbichi J-M, Yeboue K, Honde M, Diomande M, Giordano C, Doorly R, Brattegaard K, Kestens L, Smithwick RW, Kadio A, Ezani N, Yapi A, De Cock KM. The mortality and pathology of HIV disease in a west African city. AIDS 1993; 7: 1569-1579.

[9] Grant AD, Djomand G, Smets P, Kadio A, Coulibaly M, Maurice C, Whitaker JP, Sylla-Koko F, Bonard D, Wiktor SZ, Hayes RJ, De Cock KM, Greenberg AE. Profound immunosuppression across the spectrum of opportunistic disease among hospitalized HIV-infected adults in Abidjan, Cote d'Ivoire. AIDS 1997; 11: 1357-1364.

[10] Schultz TF, BoshotT CH, Weiss RA. HIV infection and neoplasia.Lancet 1996;348: 587-591.

[11] Chang Y, Cesarman E, Pessin MS, Lee F, Culpepper J, Knowles DM, Moore PS. Identification of herpesvirus-like sequences in AIDS-associated Kaposi's sarcoma. Science 1994;266(5192): 1865-1869.

[12] Price RW. Neurological complications of HIV infection. Lancet 1996;348: 445-452.

[13] Glassock R1, Cohen AH, Danovitch G, Parsa KP. Human immunodeficiency virus (HIV) infection and the kidney. Ann Intern Med 1990;112: 35-49.

[14] BHIV A Guidelines Co-ordinating Committee. British HIV Association guidelines for antiretroviral treatment of HIV seropositive individuals. Lancet 1997: 349: 1086-1092.

[15] Nunn PP, Kibuga D, Gathua SN, Brindle Rj, Imalingat A, Wasunna Kv.Lucas SB, Gilks CF, Omwega M, Were JOB, McAdams KPWJ. Cutaneous hypersensitivity reactions due to thiacetazone in HIV-1 seropositive patients treated for tuberculosis. Lancet 1991;337: 627-630. 
المظاهر السريرية المرضية لمرصى عص المناعة المكتسبة وي الكبار

\author{
سيباستيان ب. .لوكاس \\ قسم الأنسجة 1 (ضية , مستشفى القدس توماس شارع قصر لامبث , \\ لندن ، المملكة المتحلدة
}
II

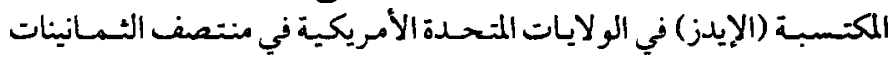

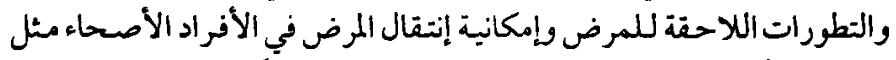

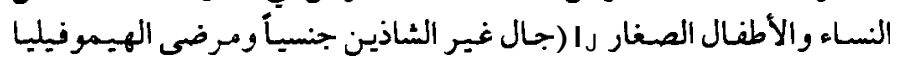

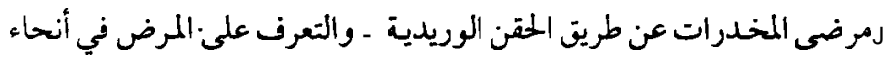

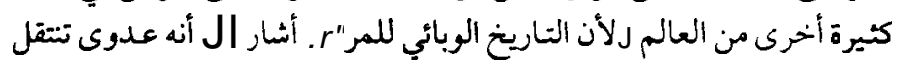

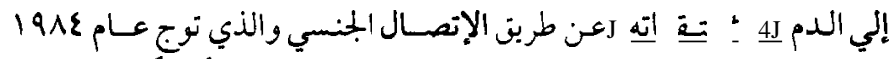

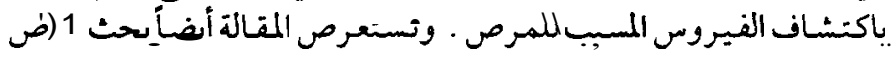

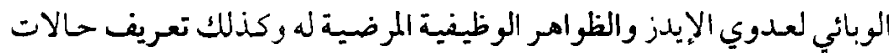

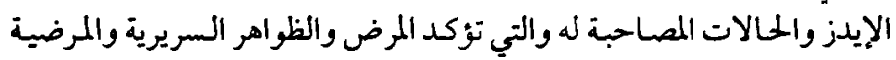

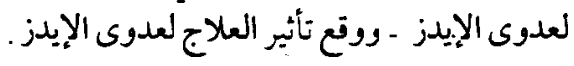

